AUDIT REPORT

\title{
UK National Audit of Early Syphilis Management. Case Notes Audit: Diagnosis and Treatment
}

\author{
Hugo McClean FRCPI, David Daniels FRCP, Chris Carne MD FRCP, Paul Bunting MSc, \\ Rob Miller FRCP
}

on behalf of the National Audit Group of the British Association for Sexual Health and HIV

Summary: A national audit of 781 early syphilis cases presenting during 2002-03 in UK genitourinary medicine clinics was conducted in late 2004, organised through the Regional Audit Groups. Data were aggregated by Region and NHS Trust, allowing practice to be compared between Regions, between Trusts within Regions, as well as to national averages and the UK National Guidelines. An enzyme immunoassay was used to diagnose 659 (89\%) cases (regional range 18-100\%). Use of a non-treponemal test was not recorded for 44 (6\%) cases. Dark ground microscopy was used in the diagnosis of only 80 (29\%) primary cases. Uptake of HIV testing was 77\% (range 69-94\%). Nationally, 527 (67\%) treatments were parenteral, with almost equal use of benzathine penicillin G for 262 (50\%, range 0-97\%) cases and procaine penicillin G (PPG) for 260 cases (49\%, range 3-100\%). There were 14 (5\%) treatments with less than the recommended $750 \mathrm{mg}$ dose of PPG. One hundred and five (40\%) PPG treatments were with greater than $750 \mathrm{mg}$ and/or for longer than 10 days of which 76 (72\%) were for early latent syphilis and/or cases with HIV infection. One hundred and ninety two (86\%, range $0-100 \%)$ of all oral treatments were with doxycycline. The recommended regimen of $100 \mathrm{mg}$ doxycycline twice daily for 14 days was used for 104 (53\%) cases; the other 91 (47\%) treatments were with a variety of regimens, mainly treatments with larger doses and/or longer treatment intervals and some combination treatments. Fourteen $(2 \%)$ cases were not treated; treatment was not reported for $7(0.9 \%)$ and not known for $10(1.3 \%)$ cases who were treated at other centres.

Keywords (MeSH): Syphilis, Syphilis Serodiagnosis, Medical Audit, Nursing Audit, Drug Therapy 


\section{Introduction}

In view of recent outbreaks of infectious syphilis in the UK, ${ }^{1}$ the National Audit Group (NAG) of the British Association for Sexual Health and HIV (BASHH) undertook an audit of early syphilis management in the UK in late 2004.

\section{Objectives \& goal}

The objectives were to provide data to allow comparison of practice against national standards and guidelines, and against average UK performances, as well as to allow comparisons between Regions and between Trusts within Regions. The goal of the audit was to improve the management of early syphilis in the UK.

\section{Methods}

The Audit methodology and organisation was planned by the NAG, involving Chairs of all the Regional Audit Groups in the UK and an NHS Trust Clinical Audit expert. The standards and guidelines used were the UK National Guidelines on the Management of Early Syphilis produced by the Clinical Effectiveness Group (CEG) of BASHH. ${ }^{2}$ These provide a series of statements about practice against which performance can be compared, including diagnosis, treatment, follow-up, contact tracing and outcomes. Consensus was reached on a final version of audit questions and two questionnaires were produced, one for audit of case note data and the other for audit of individual clinic screening and management policies. These questionnaires are available on the BASHH website. ${ }^{3}$ The audit interval was for cases first attending between 1 April 2002 and 31 March 2003.

Case definition was a case of early syphilis diagnosed within the audit interval, and case finding was based on diagnostic codes A1, A2 or A3 of the statutory KC60 and ISD(D)5 returns. ${ }^{4}$ The number of cases suggested was: single doctor clinic - first 10 cases; 2 doctors - first 20 cases; larger centres - first 30 cases during the audit interval. The following process was used to collect data. The audit questionnaires were translated into an electronic form that was emailed to Regional Chairs in the form of a Microsoft Access database. The paper questionnaires were sent to clinics in Regions by the Regional Chairs; pseudonymised data were collected by clinics on the paper questionnaires and returned to Regional Chairs. The databases were populated by the offices of Regional Chairs from the completed paper questionnaires and returned by email to a central office. These data were returned during September to December 2004, and collated and aggregated by Region and by NHS Trusts/Clinics within Regions. 


\section{Final}

$19 / 07 / 05$

This paper reports on data obtained from the case notes part of the audit with regards to demographic features of the sample, as well as performance data in terms of national and regional averages, and regional ranges, relating to the use of diagnostic tests and treatments. Data aggregated by Regions is available on the BASHH website and allows comparisons to be made between Regions. Data aggregated by NHS Trusts within Regions has been provided to Chairs of Regional Audit Groups to allow service comparisons between Trusts.

\section{Results}

Sampling and demography

Nationally, 16 of the 17 UK Regions participated, contributing a total of 781 cases made up of 702, 49 and 30 cases from England, Scotland and Northern Ireland respectively. There were a total of 2694 diagnoses of early syphilis reported during the Audit interval (personal communications - see acknowledgements), comprising 1941 primary and secondary diagnoses and 671 early latent diagnoses in England, 37 primary and secondary cases and 16 early latent diagnoses in Scotland, and 25 primary and secondary diagnoses and 4 early latent diagnoses in Northern Ireland. Hence, 27\%, 92\% and $100 \%$ of early syphilis cases in England, Scotland and Northern Ireland, respectively, were sampled. Contribution of cases from the Regions ranged from $1-15 \%$, and 255 (33\%) cases were from within the 3 London Regions. The Region outside London with the greater number of cases was the North West with $103(13 \%)$ cases. One hundred and forty four clinics took part in the Audit and 107 of these contributed cases.

Nationally, there was even distribution of cases over the age range $26-40$ years (419, 54\%); 40 $(5 \%)$ cases were aged $<20$ years and $71(9 \%)$ cases were aged $>50$ years. There were 665 (85\%, range $67-100 \%$ ) male cases and 116 (15\%, range $0-33 \%)$ female cases, of whom 26 (22\%, range $0-100 \%)$ were pregnant. There was a preponderance of female cases in the South of England with 61 (52\%) in South Thames and Anglia. Nationally 14 (54\%) pregnant women were from non-White ethnic groups.

The majority of cases were homosexual men, 465 (60\%, range 33-93\%), with heterosexual men $164(21 \%$, range $0-56 \%)$, heterosexual women 110 (14\%, range 0-33\%), bisexual men 33 (4\%, range, $0-12 \%)$; there were 3 homosexual women, and 1 bisexual woman, and sexual 
preference was not known for 5 cases. The majority were of white ethnic groups, 597 (76\%, range 56-100\%), with 154 (20\%, range 0-40\%) from other ethnic groups, comprising: Black 99 (13\%, range 0-33\%); Asian/Asian British 40 (5\%, range 0-12\%); Mixed 8 (1\%, range 0-3\%), and Chinese/Other $7(1 \%$, range $0-2 \%)$. The ethnic group was not recorded for 30 cases $(4 \%$, range $0-21 \%)$. Of 56 Black Caribbean cases, 41 (73\%) were in South London, and $23(58 \%)$ of 40 Black African cases were in South London and Anglia.

The following stages of early syphilis were reported: primary 279 (36\%, range $0-52 \%)$, secondary 250 (32\%, range $11-71 \%)$, and early latent 247 (32\%, range 4-67\%). Stage was classified as "re-infection" for one case and not recorded for 4 cases. Nationally, 477 (61\%, range 33-83\%) cases were symptomatic, with 244 (87\%, range 67-100\%) primary cases, 198 (79\%, range $0-100 \%)$ secondary cases and $33(13 \%$, range $0-100 \%)$ early latent cases having symptoms.

\section{Use of diagnostic tests}

Table 1 shows the use of diagnostic tests. The audit question about use of tests to diagnose syphilis asked whether or not particular tests were done, and data were not collected about the temporal sequence of the use of these tests. In most cases (695, 89\%, range 18-100\%) an EIA was done, with 644 (82\%, range 17-100\%) having an EIA and either TPPA or TPHA tests. The following number of negative test results/total of positive, negative, equivocal and intermediate results were reported for each test in primary syphilis cases: EIA 7/249 (2.8\%), TPPA 3/197 (1.5\%), TPHA 4/66 (6.0\%), FTA abs 4/63 (6.3\%), VDRL 40/190 (21.0\%), RPR 21/98 (21.4\%), DGM $47 / 80(58.8 \%)$. In cases of secondary syphilis, there were no negative EIA or FTA abs results, but there was one negative TPPA test, one negative TPHA test, 9 negative VDRL tests and 5 negative RPR tests. One hundred and twelve FTA abs tests were done when both an EIA and either the TPPA or TPHA tests were positive, representing $62 \%$ of the total FTA abs tests done. In no case was an EIA and FTA abs done, when the response to testing with TPPA/TPHA was not done or not answered, suggesting that the FTA abs is (appropriately) not used as a confirmatory test instead of TPPA/TPHA tests. There were $38(5 \%)$ cases where both TPPA and TPHA tests were done, although there is usually no advantage in doing both these tests. 
Use of a non-treponemal test was not recorded for $44(6 \%)$ of cases. There was highly variable use of dark ground microscopy (DGM) between Regions, and DGM was positive for around $40 \%$ of tests performed. The total of 107 DGM were performed by 32 (30\%) clinics, with 17 clinics performing one test, 4 clinics two tests, 1 clinic three tests, 2 clinics four tests, 2 clinics five tests, and seven, eight, nine, ten, thirteen and fourteen tests, one clinic each. Very little use was made either of the polymerase chain reaction (17 tests, 2 positive) or direct fluorescent antibody tests (8 tests, none positive).

Six hundred and ninety six (89\%) cases were tested for other sexually transmitted infections, and a total of 281 diagnostic codes were recorded for 220 cases, including the following: uncomplicated genital gonorrhoea (52), non-specific urethritis (43), uncomplicated genital chlamydial infection (40), first episode anogenital condylomata (18), first episode anogenital herpes simplex infection (12), viral hepatitis (6), complicated genital infection (2), trichomoniasis (5) antigen positive hepatitis B infection (1) and donovanosis (1). Table 1 shows the uptake of HIV testing; this measure included cases for whom testing was performed as well as 80 (10\%, range $0-31 \%$ ) cases already known to have HIV infection. There were 43 new diagnoses of HIV infection, most of which (32, 74\%) affected homosexual or bisexual men, although uptake of HIV testing varied by as much as $25 \%$ between Regions. Nationally, testing was declined by 130 (17\%, range $0-24 \%$ ) cases, not offered to 16 (2\%, range $0-22 \%$ ) cases, not documented for $24(3 \%$, range $0-14 \%)$ cases and this question was not answered for $9(1 \%$, range $0-6 \%)$ cases.

\section{Treatment}

Nationally, parenteral treatment was the first treatment choice for 527 (67\%, range 45-100\%) cases, with approximately equal use of benzathine penicillin $\mathrm{G}$ (BPG) and procaine penicillin $\mathrm{G}$ (PPG) (including 11 Bicillin treatments) for 262 (50\%, 0-97\%) and 260 (49\%, 3-100\%) cases respectively (Table 2). An oral treatment was the first treatment choice for $223(28 \%$, range 0 $52 \%$ ) cases (Table 3), with doxycycline as the only oral treatment comprising 192 (86\%, range $50-100 \%$ ) of oral treatments (Table 4$)$. Fourteen cases (2\%, range $0-6 \%$ ) were not treated. Ten $(2 \%$, range $0-11 \%)$ cases were treated by another clinic and treatment was not known, and no report of treatment was received for the remaining 7 (1\%, range $0-6 \%)$ cases.

The most common dose of PPG was with the 750mg dose (Table 2). Sixty seven (26\%) PPG treatments were with doses $>750 \mathrm{mg}$, comprising 2 grams (19 treatments), 0.9 grams (12 


\section{Final}

treatments), 0.8 grams (7 treatments), 1.2 grams (7 treatments), 2.4 grams ( 7 treatments), 2.67 grams (one treatment), 1.8 grams (one treatment), Jenacillin $A 3.6 \mathrm{ml}$ (2 treatments), one treatment each of Jenacillin $A 4 \mathrm{ml}, 7.2$ and $8 \mathrm{ml}$, one treatment with Jenacillin (not specified) $4 \mathrm{ml}$, Bicillin 1.2 grams (2 treatments), Bicillin $3 \mathrm{ml}$ (one treatment), and Bicillin 0.8 grams (4 treatments). Of these, only 28 (42\%) were treatments for early latent syphilis, and only 27 (40\%) were treatments for cases with HIV infection.

In $14(5 \%)$ instances treatment was with a dose <750mg PPG, comprising Jenacillin A 2.4ml (6 treatments), PPG 0.6 grams (5 treatments), Jenacillin (not specified) $2.4 \mathrm{mls}$ (2 treatments), and $250 \mathrm{mg}$ (one treatment). There were $5(2 \%)$ treatments with long-acting PPG 2 grams. The dose used was not clear for $11(4 \%)$ treatments. In one Region amoxycillin plus probenecid was used for 3 cases for weekend treatment to substitute for PPG.

Ninety four (36\%) PPG treatments were for $>10$ days: 10 (4\%) cases were treated for 12 days, $37(14 \%)$ for 14 days, $1(0.5 \%)$ for 15 days, $39(15 \%)$ for 17 days, $2(1 \%)$ for 19 days, $1(0.5 \%)$ for 20 days, 4 (2\%) for 21 days and not specified for 7 (3\%). Of cases treated for longer than 10 days, only $46(29 \%)$ were treatments for early latent syphilis and only $36(23 \%)$ were treatments for cases with HIV infection. Nationally, 105 (40\%) PPG treatments (of cases in 33 clinics in 14 Regions) were with greater than $750 \mathrm{mg}$ and/or for longer than 10 days; $49(47 \%)$ of these treatments were for early latent syphilis, 37 (35\%) for cases with HIV infection, and 76 (72\%) for early latent syphilis and/or cases with HIV infection.

Doxycycline was used to treat 195 (25\%) cases, including three treatments combined with azithromycin. The recommended regimen of $100 \mathrm{mg}$ twice daily $(\mathrm{bd})$ for 14 days was used in $104(53 \%)$ cases. However, 91 (47\%) treatments were with a variety of other regimens, including treatment with larger doses and/or longer treatment intervals, once daily treatment and some combination treatments. Of these, $75(82 \%)$ bd treatments were with $>100 \mathrm{mg}$ bd and/or for longer than 14 days; 33 (44\%) of these treatments were for early latent syphilis, $18(24 \%)$ for cases with HIV infection, and $46(61 \%)$ for early latent syphilis and/or cases with HIV infection. The remaining $16(18 \%)$ treatments were with a variety of once daily regimens (Table 4).

Of 123 cases who also had HIV infection, $88(72 \%$, range $0-100 \%)$ and $31(25 \%$, range 0 $100 \%)$ received parenteral and oral treatments respectively, as treatments of first choice; $2(2 \%)$ cases were treated elsewhere and treatment was not known, treatment was not recorded for 


\section{Final}

$19 / 07 / 05$

one $(1 \%)$ case and one $(1 \%)$ case was not treated. Of the parenteral treatments used to treat cases with HIV infection, 56 (64\%) were with PPG, 15 (17\%) with 2 dose of BPG, 9 (10\%) with 1 dose of BPG, $6(7 \%)$ with 3 doses of BPG, one (1\%) with benzyl penicillin $G$, and one $(1 \%)$ with ceftriaxone. Of the 56 treatments with PPG, 15 (27\%) were for 10 days, $2(4 \%)$ for 12 days, $6(11 \%)$ for 14 days, 1 (2\%) for 15 days, $25(45 \%)$ for 17 days, 1 (2\%) for 19 days, $1(2 \%)$ for 21 days, and not stated for $5(9 \%)$. A total of $27(22 \%)$ cases with HIV infection were treated with doxycycline.

There were 25 treatments involving the use of probenecid, 13 of 15 treatments with amoxycillin (87\%), and 12 treatments with PPG (including one treatment with Bicillin). Of the latter, 5 treatments were for early latent syphilis (all of these were with doses $>750 \mathrm{mg}$ for 17-21 days), 5 treatments for secondary syphilis (also with doses $>750 \mathrm{mg}$ for $14-24$ days), and the remaining two treatments were for primary syphilis, one with 2 grams of PPG for 17 days.

There were 29 repeat treatments; in three cases re-infection was reported, and no cases of relapse or treatment failure were reported. Eleven initial treatments with PPG were repeated: 6 with doxycycline treatments, two with erythromycin 500mg twice daily for 14 days, one with azithromycin one gram stat, one with two doses of BPG, and the remaining re-treatment was not specified. Nine initial treatments with BPG were repeated: 6 treatments with doxycycline and 3 treatments with PPG. Six initial treatments with doxycycline were repeated: three with BPG, two with higher dose doxycycline regimen and one with azithromycin 500mg PO daily for 10 days. Two initial treatments with a stat dose of azithromycin were each repeated with azithromycin 500mg once daily for 10 days. One ceftriaxone treatment was repeated with a course of erythromycin treatment.

Nationally, 509 (65\%, range 43-100\%) treatments were directly observed, but these were nearly all parenteral treatments: 503 of $527(95 \%)$ parenteral treatments were reported as directly observed. The remaining six cases comprised 5 oral treatments ( 1 for a case with HIV infection) and an unknown treatment.

Advice about adverse drug reactions was received by 516 (66\%, range $0-87 \%)$ cases. There were a total of 50 (6\%, range $0-23 \%)$ cases affected by adverse events, comprising $35(7 \%)$ and $15(7 \%)$ cases receiving 527 parenteral and 223 oral treatments respectively. Fourteen of the oral treatment events were with doxycycline, and one with amoxycillin. The following 50 


\section{Final}

adverse reactions affecting 48 cases were reported: Jarish-Herxheimer reaction (12), rash (11), paraesthesia/pain symptoms (6), fever (4), fainting (3), nausea (3), vomiting (3), diarrhoea (2), flu-like symptoms (1), lower limb oedema (1), panic attack (1), procaine reaction (1), pruritis (1), weight loss (1) and 3 non-specified events; there was no description of the adverse reactions affecting two cases. A course of corticosteroids was prescribed for 33 cases $(4 \%$, range $0-14 \%$, in 10 Regions). The indications for use of corticosteroids were as follows: specific prevention of the Jarish-Herxheimer reaction (12), treatment of the Jarish-Herxheimer reaction (3), prophylactic use (3), treatment of a possible Jarish-Herxheimer reaction (1), treatment of choroidretinitis (1) and treatment of cranial nerve palsy (1). In 12 cases no information was presented to explain the use of corticosteroids. Three corticosteroid treatments were during pregnancy - one for possible Jarish-Herxheimer reaction, and not specified for the other two cases.

\section{Discussion}

This is the first UK national audit of early syphilis management, and the first national audit to provide clinical performance data aggregated by Regions and NHS Trusts. Over one quarter of cases in England and most cases in Scotland and Northern Ireland were sampled. Data has rapidly been disseminated to Chairs of Regional Audit Groups to facilitate comparative and interventional work that might lead to maintaining or improving practice.

The absence of symptoms in some cases of primary and secondary syphilis, reporting of symptoms in some cases of early latent syphilis, as well as use of higher doses and longer durations of PPG regimens, suggests unsatisfactory use of diagnostic codes for case definition. However, it is possible that some treatments of primary syphilis pre-empted, or took place before, the appearance of symptoms (e.g. where epidemiological treatment took place).

The PHLS Syphilis Serology Working Group ${ }^{5}$ recommended that use of the FTA abs test is reserved for discrepant results between EIA and TPPA/TPHA tests, although this recommendation is not directly referred to in the CEG Guidelines. ${ }^{2}$ The observation that $62 \%$ of FTA abs tests were carried out when both an EIA and either the TPPA or TPHA were positive suggests either frequent use of the FTA abs test for very early diagnosis, or overuse of the FTA abs test for confirmation. The much greater use of the TPPA test compared to the TPHA test 
probably reflects the greater laboratory convenience of use of the former test. It is noteworthy that the commonly used tests do not have $100 \%$ sensitivity in primary syphilis, emphasising the importance of repeat testing. DGM was demonstrated to be a useful diagnostic tool in this audit with positivity rates of up to $100 \%$. A survey conducted by the British Co-operative Clinical Group (BCCG) of BASSH in 2002 has, however, shown that $35 \%$ of clinics were not confident of their ability to detect treponemes on DGM. ${ }^{6}$ The very low use of the two other main direct detection methods, PCR and DFA, probably reflects lack of data about the relative contribution of these tests to earlier and/or increased detection of early syphilis. DGM is relatively easier to provide than PCR and DFA. This Audit supports the recommendation of a recent evaluation, ${ }^{7}$ clearly showing the usefulness of DGM in the early diagnosis of primary and secondary syphilis and that DGM should be available in all genitourinary medicine clinics.

There was an important number of cases who received treatment doses and or durations greater than those recommended by the CEG guidelines. This includes $38 \%$ of doxycycline bd treatments with $>100 \mathrm{mg}$ bd and/or for longer than 14 days, and $40 \%$ of PPG treatments with greater than $750 \mathrm{mg}$ and/or for longer than 10 days. Treatments in excess of the recommended doxycycline regimen in early syphilis may reflect concern about reported treatment failure with the recommended regimen for doxycycline, ${ }^{8}$ treatment modifications because of drug interactions, concern about possible asymptomatic neurosyphilis, or the weak evidence base supporting the currently recommended doxycycline treatment for early syphilis. The once daily treatments with doxycycline may reflect treatment simplification to encourage adherence. Most use of higher doses and longer durations of PPG regimens was found in cases with early latent syphilis or with HIV infection, although $28 \%$ of PPG treatments in excess of those recommended by the National Guidelines were for neither early latent syphilis nor HIV infection and might be explained by concern about possible asymptomatic neurosyphilis. By contrast, based on the recommendation in the National Guidelines that cases with HIV infection are treated "as for neurosyphilis," there is considerable under-treatment of early syphilis in cases with HIV infection. ${ }^{2}$

\section{Conclusions and suggested areas for practice improvement/intervention}

Increased use of pre-treatment non-treponemal tests to $100 \%$ is needed in some Regions. More use should be made of DGM; training is available in the UK to support increased use of DGM (details available on the BASHH website). HIV infection is particularly important in early syphilis 
and some Regions should consider interventions to improve uptake of HIV testing. More research is needed to determine the role of direct detection methods in early syphilis diagnosis.

There was a considerable amount of treatment in excess of that recommended by the UK National Guidelines for early syphilis management, not all attributable to treatment of cases with HIV infection. This suggests inappropriate use of diagnostic codes or, particularly with regard to doxycycline treatments, lack of confidence among clinicians in the currently recommended treatments. Further work may be needed to improve the definition or appropriate use of the early syphilis diagnostic codes. Alternatively, increased stakeholder involvement in the writing of the UK guidelines for early syphilis management, such as that described in a recent article on guideline development, ${ }^{9}$ may improve use of recommended treatments. Based on the National Guidelines $^{2}$ there is considerable under-treatment of early syphilis in cases with HIV infection that may improve with the publication of guidelines on treatment of acquired syphilis in HIV infection. ${ }^{10}$

Detailed data, aggregated by Region, are available on the BASHH website: $h$ ttp://www.bashh.org/committees/nag/index.htm

The work done by the Regional Chairs and staff in NHS Trusts/Clinics is gratefully acknowledged:

Anglia: Dr. Kanagarat Sivakumar, Gaye Omara, Addenbrooke's Hospital; Bedford Hospital NHS Trust; Hinchingbrooke Hospital; James Paget Healthcare NHS Trust; Luton \& Dunstable; Norfolk and Norwich; Peterborough and Stamford; The Queen Elizabeth; West Suffolk. Essex: Dr. Sam Jebakumar on behalf of The Essex Interdistrict Audit Group: Essex County Hospital; Princess Alexandra NHS Trust; St Johns Mid Essex Trust; Thurrock PCT. Merseyside: Dr. Ravindra Gokhale, Countess of Chester Hospital NHS Trust; Midcheshire Hospital NHS Trust; North Cheshire Hospital NHS Trust; Royal Liverpool; St Helens \& Knowsley NHS Trust; Wirral Hospital NHS Trust. North Thames: Dr. David Daniels; Dr. Rob Miller, Karen Jones on behalf of the North Thames GUM/HIV Audit Group: Archway Sexual Health Clinic; Barking, Havering \& Redbridge Hospitals NHS Trust; Barts \& The London Hospitals NHS Trust; Camden PCT; Central Middlesex Hospital; Charing Cross Hospital; Chelsea \& Westminster Hospital; Victoria Clinic; Ealing Hospital; Essex County Hospital; Haringey PCT; Homerton Hospital; Lister Hospital; Newham General Hospital; Northwick Park Hospital; Princess Alexandra NHS Trust; South East Herts PCT; Southend General Hospital; St Mary's Hospital; West Middlesex Hospital. North West: Dr. Ashish Sukthankar, on behalf of North West BASHH Regional Audit Group: Victoria Hospital, Blackpool Primary Care Trust; Manchester Royal Infirmary, Central Manchester and Manchester Children's University Hospitals NHS Trust; Blackburn Royal Infirmary, East Lancashire Hospitals NHS Trust; North Manchester General Hospital, The Pennine Acute Hospitals NHS Trust; Fairfield General Hospital, Bury, The Pennine Acute Hospitals NHS Trust; The Bridge Sexual Health Centre, Rochdale, The Pennine Acute Hospitals NHS Trust; Royal Preston Hospital, Preston Acute Hospitals NHS Trust; Hope Hospital, Salford Royal Hospitals NHS Trust; Withington Hospital, South Manchester University Hospital NHS Trust; Centre for Sexual Health, Tameside \& Glossop Primary Care Trust; Royal Albert Edward Infirmary, Wrightington, Wigan \& Leigh NHS Trust. Northern: Dr. Conrad White: 1 to 1 centre; North Shields; Bishop Auckland General Hospital; Darlington Memorial Hospital; James Cook University Hospitals Middlesborough; Newcastle General Hospital; North Cumbria Acute NHS Hospitals; South Tyneside PCT; Sunderland Royal Hospital; University Hospital of Hartlepool; University Hospital of North Durham; Northern Ireland: Dr.Raymond Maw, Dr. Ciara Cunningham, Dr. Carol Emerson, Joe Ifediora: Royal Group Hospitals, Belfast. Oxford: Dr. Graz Luzzi, Carol Bingham on behalf of: Buckinghamshire Hospitals NHS Trust; Northamptonshire Healthcare NHS Trust; Oxford Radcliffe NHS Trust; Royal Berkshire and Battle Hospitals NHS Trust and Slough PCT. Scotland: Dr Steve Baguley, Lesley Gallagher, on behalf of Scottish Branch of BASHH: Woolmanhill Hospital, Aberdeen; Orchard House Health Centre, Stirling; Borders Sexual Health Service, Borders General Hospital, Melrose; Russell Institute, Paisley; Ninewells Hospital, Dundee; Sandyford Initiative Glasgow; Highland Sexual Health, Raigmore Hospital, Inverness; Royal Infirmary of Edinburgh; Heathfield Hospital, Ayr; 
Monklands Hospital, Airdrie. South East Thames: Dr. Joseph Winceslaus; Dr Emma Fox; Paul Bunting; Avenue House Clinic, Eastborne; Beckenham Hospital, Bromley; Darent Valley Hospital, Dartford; Guy's \& St Thomas' Trust, London; Kent \& Sussex Hospital, Tunbridge Wells; King's College Hospital, London; Ore Clinic, Hastings; Preston Hall Hospital, Maidstone; Queen Elizabeth Hospital, Woolwich; Royal Sussex County Hospital, Brighton. South West: Dr. Zubayr Sulaiman, Sarah Norman, on behalf of South West GU Physicians Group/Branch of BASHH: Gloucestershire Royal \& Cheltenham General Hospitals; Northern Devon Healthcare NHS Trust; Plymouth Hospital NHS Trust (Derriford); Royal Cornwall Hospitals Trust; Royal Devon \& Exeter Healthcare NHS Trust; Royal United Hospital Bath NHS Trust. South West Thames: Dr.Tony Newell; Dr Steven Estreich; Paul.Bunting on behalf of the South Thames GU Medicine Audit Group: Crawley Hospital; East Surrey Hospital, Redhill; Farnham Road Hospital, Guildford; Frimley Park Hospital, Camberley; Kingston Hospital, Kingston-uponThames; Leatherhead Hospital; Mayday University Hospital, Croydon; Queen Mary's Hospital, Roehampton, London; St George's Hospital, London; St Helier Hospital, Carshalton; St Peter's Hospital, Chertsey; Southlands Hospital, Shoreham by Sea. Trent: Dr. Jyoti Dhar on behalf of the Trent Region Genitourinary Medicine Audit Group: Barnsley District General Hospital NHS Trust; Charnwood and Northwest Leicestershire; Kings Mill Hospital; Leicester University Hospitals; Grimsby Health NHS Trust; Nottingham City Hospital NHS Trust; Rotherham General Hospitals NHS Trust; Sheffield Teaching Hospitals NHS Trust; Southern Derbyshire Acute Hospitals NHS Trust. Wessex: Linda Tucker Nurse Consultant on behalf of Wessex Regional Audit Group: Andover War Memorial Community Hospital; The Royal Bournemouth \& Christchurch Hospitals NHS Trust; Isle of Wight Healthcare NHS Trust; Portsmouth Hospitals NHS Trust; Royal Hampshire County Hospital; Royal West Sussex County Hospital; Salisbury Health Care NHS Trust; Southampton University Hospitals NHS Trust; West Dorset General Hospitals NHS Trust; Winchester \& Eastleigh Hospitals NHS Trust. West Midlands: Dr Mia Huengsberg on behalf of West Midlands Regional Audit Committee: Alexandria Hospital, Redditch; George Eliot Hospital, Nuneaton; Heartlands Hospital, Birmingham; Princeton Royal Hospital, Telford; Queens Hospital, Burtonon-Trent; Royal Shrewsbury Hospital; South Warwickshire PCT; South Worcestershire PCT; University Hospital North Staffordshire, Stoke-on-Trent; Walsgrave Hospital NHS Trust, Coventry; Whittall Street Clinic, Birmingham. Yorkshire: Dr.Hugo McClean, Dr. Nicola Armstrong, Claire Thurston on behalf of the Yorkshire Multi-district Clinical Audit Group: Calderdale \& Huddersfield Acute NHS Trust; Castle Hill Hospital, Cottingham; Leeds Teaching Hospital; Northern Lincolnshire \& Goole Hospitals NHS Trust; The Mid Yorkshire Hospitals NHS Trust; West Hull PCT; York Hospitals NHS Trust.

Stephen Walker, Senior Information Officer, Research \& Development, Humber Mental Health Trust for technical support with the database. Michael Toorie, Health Protection Agency, Communicable Disease Surveillance Centre, for providing the sample denominator for England \& Northern Ireland, Dr Lesley A Wallace, Scottish Centre for Infection and Environmental Health, Health Protection Scotland, for providing the sample denominator for Scotland.

Correspondence to: Dr. Hugo McClean, Sexual Health Resource Centre, Conifer House, West Hull Primary Care NHS Trust, Conifer House, 32-36 Prospect Street, Hull, HU2 8PX. E-mail: Hugo.McClean@whpct.nhs.uk

Brown AE, KE Sadler, SE Tomkins et al. Recent trends in HIV and other STIs in the United Kingdom: data to the end of 2002. Sex Transm Infect 2004;80:159-66 British Association for Sexual Health and HIV. Clinical Effectiveness Group. UK National Guidelines on the Management of Early Syphilis 2002 [www.bashh.org/guidelines/2002/early\$final0502.pdf] (last accessed 1 May 2005) British Association for Sexual Health and HIV. National Audit Group. Draft Clinical Practice Questionnaires and Audit Proformas: early syphilis case-notes.

[www.bashh.org/committees/nag/questionnaires/nag_early_syphilis\%20case-notes_v2.doc] (last accessed 1 May 2005)

PHLS, DHSS\&PS and the Scottish ISD(D)5 Collaborative Group. Sexually Transmitted in the UK: New Episodes seen at Genitourinary Medicine Clinics 1991-2001. London: Public Health Laboratory Services, 2002 
5 Egglestone SI, Turner AJL, for the PHLS Syphilis Working Group. Serological diagnosis of syphilis. Commun Dis Public Health 2000; 3:158-62

6 Rogstad KE, Simms I, Fenton KA, Edwards S, Fisher M, Carne CA. Screening, Diagnosis, and Management of Early Syphilis in Genitourinary Medicine Clinics in the United Kingdom. Int J STD AIDS 2005;16:348-52

7 Wheeler HL, Agarwal S, Goh BT. Dark ground microscopy and treponemal serological tests in the diagnosis of early syphilis. Sex Transm Infect 2004; 80: 411 - 414

8 Goorney B, Leahy M. Relapse of early syphilis on first line treatment. Int J STD AIDS. 2002;13(10):722-3

9 Rooney G, Cluzeau FA, Daniels D, et al. Getting the guidance right: optimizing the quality of the UK national guidelines on sexually transmitted infections and closely related conditions. Int J STD AIDS 2004; 15: 297-298

Nandwani R, Fisher M on behalf of the MSSVD HIV Special Interest Group. Clinical standards for the screening and management of acquired syphilis in HIV-positive adults. London (UK): Medical Society for the Study of Venereal Diseases (MSSVD); 2002. US National Guideline Clearinghouse: NGC 002666 [www.guideline.gov/summary/summary.aspx?ss=15\&doc_id=3440\&nbr=2666\&string=syphilis\%20AND\%2 OHIV]. Also available at www.bashh.org/guidelines/ceguidelines.htm. (Last accessed 12 July 2005)

Table 1. Use of diagnostic tests in early syphilis ( $n=781$ unless otherwise stated)

\begin{tabular}{lrrr}
\hline \multicolumn{1}{c}{ Test } & $\begin{array}{c}\text { Number of tests } \\
\text { done (\%) }\end{array}$ & $\begin{array}{c}\text { Regional } \\
\text { ranges (\%) }\end{array}$ & $\begin{array}{c}\text { Percentage } \\
\text { positive tests } \\
\text { (regional range) }\end{array}$ \\
\hline Treponemal enzyme immunoassay (EIA) & $695(89)$ & $18-100$ & $98(89-100)$ \\
Treponema pallidum particle agglutination assay (TPPA) & $524(67)$ & $1-100$ & $99(89-100)$ \\
Treponema pallidum haemagglutination assay (TPHA) & $229(28)$ & $0-98$ & $96(78-100)$ \\
Fluorescent treponemal antibody absorption test (FTA abs) & $182(23)$ & $0-78$ & $91(40-100)$ \\
Venereal Diseases Research Laboratory (VDRL) & $531(68)$ & $4-100$ & $80(0-100)$ \\
Rapid plasma reagin test (RPR) & $298(38)$ & $0-100$ & $85(50-100)$ \\
VDRL or RPR & $737(94)$ & $50-100$ & $84(44-96)$ \\
Direct fluorescent antibody (DFA) & $8(1)$ & $0-5$ & $0(0)$ \\
Polymerase chain reaction (PCR) & $18(2)$ & $0-6$ & $11(0-100)$ \\
Dark ground microscopy (all cases) & $107(14)$ & $0-40$ & $37(0-100)$ \\
Dark ground microscopy (primary syphilis, $\mathrm{n}=279)$ & $80(29)$ & $0-82$ & $41(0-100)$ \\
Screening for other STls & $696(89)$ & $76-97$ & See text \\
HIV testing - uptake & $602(77)$ & $69-94$ & $6(0-10)^{*}$ \\
Pregnancy testing ( $\mathrm{n}=116)$ & $18(16)$ & $0-53$ & Not audited \\
\hline
\end{tabular}

*Newly diagnosed HIV infection only, denominator is total cases minus previously diagnosed cases 
Table 2. Initial parenteral treatments for early syphilis $(n=527)$

\begin{tabular}{lrr}
\hline \multicolumn{1}{c}{ Parenteral treatment } & $\begin{array}{r}\text { Number of } \\
\text { treatments }(\%)\end{array}$ & $\begin{array}{c}\text { Regional } \\
\text { ranges \% }\end{array}$ \\
\hline Procaine penicillin G & $260(49)$ & $3-100$ \\
Use of 750mg dose* & $163(63)$ & $32-100$ \\
$\quad$ Treatment for 10 days & $159(61)$ & $36-100$ \\
Benzathine penicillin G: 2 doses & $188(36)$ & $0-93$ \\
Benzathine penicillin G: 1 dose & $62(12)$ & $0-39$ \\
Benzathine penicillin G: 3 doses & $12(2)$ & $0-20$ \\
Ceftriaxone & $3(0.6)$ & $0-4$ \\
Benzyl penicillin & $2(0.4)$ & $0-3$ \\
\hline
\end{tabular}

*Includes 11 treatments with Bicillin

†750mg, 750,000IU, Jenacillin A 3.3ml, Jenacillin A 3ml and Jenacillin (not specified) 3mls were counted in as $750 \mathrm{mg}$ doses of procaine penicillin $\mathrm{G}$

Table 3. Initial oral treatments for early syphilis $(n=223)$

\begin{tabular}{lrr}
\hline \multicolumn{1}{c}{ Oral treatment } & $\begin{array}{c}\text { Number of } \\
\text { treatments }(\%)\end{array}$ & $\begin{array}{r}\text { Regional ranges } \\
\%\end{array}$ \\
\hline Doxycycline $^{*}$ & $192(86)$ & $50-100$ \\
$\quad$ Use of 100mg twice daily 14 days regimen & $104(54)$ & $0-100$ \\
Amoxycillin† & $15(7)$ & $0-20$ \\
Azithromycin & $8(4)$ & $0-25$ \\
Erythromycin & $4(2)$ & $0-11$ \\
Azithromycin plus doxycycline & $3(1)$ & $0-50$ \\
Oxytetracycline & $1(0.5)$ & $0-7$ \\
\hline *3 doxycycline treatments combined with azithromycin excluded & & \\
†2 treatments without probenecid & &
\end{tabular}


Table 4. Initial treatments with doxycycline for early syphilis $(n=195)$

\section{Doxycycline treatment}

Number of treatments (\%)

Doxycycline $100 \mathrm{mg}$ bd for 14 days

Doxycycline $100 \mathrm{mg}$ bd for 14 days plus benzathine penicillin 2.4 grams x $1 \mathrm{IM}$ $103(53)$

Doxycycline $200 \mathrm{mg}$ bd for 28 days

Doxycycline $200 \mathrm{mg}$ bd for 14 days

Doxycycline $100 \mathrm{mg}$ bd for 28 days

Doxycycline $300 \mathrm{mg}$ od for 14 days

Doxycycline $100 \mathrm{mg}$ bd for 21 days

Doxycycline $100 \mathrm{mg}$ tds for 21 days

Doxycycline $200 \mathrm{mg}$ bd for 21 days

Doxycycline $200 \mathrm{mg}$ od for 14 days

Doxycycline $300 \mathrm{mg}$ od for 21 days

Doxycycline $100 \mathrm{mg}$ bd for 20 days

Doxycycline $100 \mathrm{mg}$ tds for 28 days

Doxycycline $100 \mathrm{mg}$ od for 21 days

Doxycycline $100 \mathrm{mg}$ for 21 days (dosing not specified)

Doxycycline $100 \mathrm{mg}$ tds for 14 days

Doxycycline $100 \mathrm{mg}$ mane, $200 \mathrm{mg}$ nocte 14 days

Doxycycline $100 \mathrm{mg}$ bd for 30 days

Doxycycline $200 \mathrm{mg}$ mane, $100 \mathrm{mg}$ nocte for 21 days

Doxycycline $200 \mathrm{mg}$ tds for 21 days

Doxycycline $100 \mathrm{mg}$ tds 14 days plus azithromycin $1 \mathrm{~g}$ stat

\section{END.}

